Econ. Theory 5, 337-351 (1995)

Economic
Theory

(C) Springer-Verlag 1995

\title{
Equilibria in a multi-object uniform price sealed bid auction with multi-unit demands *
}

\author{
Charles Noussair \\ Econometric Institute, Erasmus University Rotterdam, Postbus 1738, 50 Burg, Oudlaan, 3000 DR \\ Rotterdam, THE NETHERLANDS
}

Received: September 30, 1992; revised version November 22, 1993

\begin{abstract}
Summary. In many existing markets demanders wish to buy more than one unit from a group of identical units of a commodity. Often, the units are sold simultaneously by auction. The vast majority of literature pertaining to the economics of auctions, however, considers environments in which demanders buy at most one object. In this paper we derive necessary and sufficient conditions for a set of bidding strategies to be a symmetric monotone Bayes-Nash equilibrium to a uniform price sealed bid auction using the "first rejected bid pricing rule" in an independent private values environment with two-unit demands. In any symmetric monotone BayesNash equilibrium, all bidders submit one bid equal to their higher valuation and one bid lower than their lower valuation. We characterize the equilibrium and derive the exact amount of underrevelation in the lower bid.
\end{abstract}

\section{Introduction}

Auctions are used in every part of the world to transact trillions of dollars worth of objects every year. The omnipresence of auctions has certainly not gone unnoticed by economists who have generated a huge literature on the subject. Most of the literature focuses on environments where a single seller has one or more indivisible object(s) to be sold to multiple bidders, each of whom wants to buy at most one of the objects. In the first major paper on the subject, Vickrey (1961) introduces the second price sealed bid auction and its multi-unit generalization, the uniform price sealed bid auction with "first rejected bid" pricing. In an environment in which each demander independently draws one valuation, known only to her, from a distribution which is known to all demanders, these auctions

\footnotetext{
* This article is based on chapter 2 of the author's $\mathrm{Ph}$.D. thesis. I would like to acknowledge the financial support of the Clarence Hicks Memorial Fellowship, the California Institute of Technology and the Jet Propulsion Laboratory. I also thank D. Roderick Kiewiet, Richard McKelvey, Charles Plott, David Porter, Annemieke Tromp, participants in the Tinbergen Institute Seminar Series, an anonymous referee and especially John Ledyard for countless enlightening comments.
} 
are demand revealing. Each bidder has a dominant strategy to submit a bid equal to the value she has drawn. ${ }^{1}$

In most markets where auctions are currently used, however, it is common for buyers to wish to buy more than one unit of the commodity. If a demander wishes to and is permitted to purchase more than one unit, non-uniform pricing is required to induce demand revelation (Vickrey (1961), Forsythe and Isaac (1982), Weber (1983)). ${ }^{2}$ The demand revealing auctions are quite complex and in many applications the simplicity of a uniform pricing rule may be preferred or required. Assessing the consequences of using a uniform price auction and the resulting strategic behavior on the part of bidders requires an analysis of the equilibrium properties of the particular auction applied. Characterization of equilibria for a simple uniform price auction is thus the focus of this paper.

In the next section we model a uniform price sealed bid auction with "first rejected bid" pricing in an independent private values environment with two-unit demands. In theorem 1, we list necessary conditions for a bidding rule to be a symmetric undominated strictly monotone Bayes-Nash equilibrium. In equilibrium, each demander bids his valuation for his higher-valued unit and less than his valuation for his lower-valued unit. The exact amount of underrevelation is derived in lemma 2 . The bidding function must be separable, in the sense that each bidder's lower bid is independent of his higher valuation and vice-versa. In theorem 2, sufficient conditions for a bidding function satisfying the conditions of theorem 1 to be an equilibrium are given.

\section{The uniform price sealed bid auction}

In this section we consider the theoretical properties of a simple uniform price sealed bid auction within a two-unit demand independent private values environment. Necessary and sufficient conditions for a bidding function to be a symmetric monotone Bayes-Nash equilibrium are derived, and an example is provided.

\subsection{The model}

Let there be $k(>1)$ identical units to be sold and $n+1(>1)$ demanders indexed by $i=1, \ldots, n+1$. Each demander draws two valuations independently from a fixed and common distribution $\gamma(v)$, where $\gamma(v)$ has strictly positive density on $[0, \bar{v}] \subset R^{+}$ and $\gamma(v) \in C^{2}$. Order the two values from higher to lower and index them 1 and 2 respectively, so that $v_{1}^{i} \geq v_{2}^{i} \geq 0$ are the valuations of demander $i$. Define $G\left(v_{1}^{i}, v_{2}^{i}\right)=$ $\operatorname{Prob}\left(v_{1} \leq v_{1}^{i}, v_{2} \leq v_{2}^{i}\right)$, where $v_{1}$ and $v_{2}$ are a pair of values independently drawn from $\gamma(v)$. Let $g\left(v_{1}, v_{2}\right)$ denote the probability density function of $G$. Since $g$ is a joint density of order statistics drawn from a distribution with positive density on $[0, \bar{v}]$, $g\left(v_{1}, v_{2}\right)>0$ for all $v_{1}, v_{2}$ such that $0 \leq v_{2} \leq v_{1} \leq \bar{v}$. All demanders are risk neutral. Valuations are private information but $\gamma, n$ and $k$ are common knowledge.

\footnotetext{
${ }^{1}$ Forsythe and Isaac (1982) show that the second price auction is the only demand revealing direct mechanism in the single-unit environment.

${ }^{2}$ All of the demand revealing auctions are variations of Groves mechanisms.
} 


\subsection{The game}

All demanders submit two non-negative bids. The highest $k$ bids are accepted and the corresponding demanders pay a per-unit price equal to the $k+1$ st highest bid. A tie for $k$ th highest bid is broken by randomly allocating a unit to one of the tied demanders. A bid which is equal to zero is never accepted.

\section{Symmetric equilibria}

\subsection{Necessary conditions}

In theorem 1 we derive a necessary condition for a bidding strategy to be a symmetric monotone Bayes-Nash equilibrium. Let a bidding function, $B\left(v_{1}, v_{2}\right)=$ $\left(B_{1}\left(v_{1}, v_{2}\right), B_{2}\left(v_{1}, v_{2}\right)\right):[0, \bar{v}]^{2} \rightarrow R^{2+}$, map two valuations into two bids. Two definitions are required for the statement and proof of the theorem.

Definition 1. $A$ bidding function, $B\left(v_{1}, v_{2}\right)$ is type $M$ if:

1) $B(0,0)=(0,0)$,

2) $B$ is continuous in $v_{1}$ and $v_{2}$,

3) $B_{1}(\bar{v}, \bar{v}) \geq B_{2}(\bar{v}, \bar{v})$,

4) $\exists$ a function $\hat{v}_{j}\left(v_{z}\right)$ such that $B_{j}\left(v_{z}, v_{j}\right)=0$; iff $v_{j} \leq \hat{v}_{j}\left(v_{z}\right)$, for $z \neq j ; z, j \in 1,2$.

5) $\frac{\partial B_{j}}{\partial v_{j}}$ exists and is $>0$ if $B_{j}>0$ and $\frac{\partial B_{j}}{\partial v_{z}}$ exists and is $\geq 0 ; z \neq j$ if $B_{j}>0$.

Definition 1 describes a notion of continuous monotonicity. $B_{1}$ and $B_{2}$ are monotone in both of their arguments and strictly monotone in one of them. However, 1) and 3) impose restrictions on the bidding function which make type $M$ differ from more standard notions of monotonicity. The type $M$ class allows bidding strategies which specify that $B_{j}\left(v_{1}, v_{2}\right)=0$ for all $v_{1} \leq \hat{v}_{1}$ and $v_{2} \leq \hat{v}_{2}$, for any $\hat{v}_{1} \in[0, \bar{v}]$ and any $\hat{v}_{2} \in[0, \bar{v}]$. It includes, as a special case, bidding functions which are strictly increasing in $v_{1}$ and $v_{2}$ (where $0=\hat{v}_{2}=\hat{v}_{1}$ ). The important concept of separability is described in definition 2 .

Definition 2. $A$ bidding function is separable if $B\left(v_{1}, v_{2}\right)=\left(B_{1}\left(v_{1}\right), B_{2}\left(v_{2}\right)\right)$. That is, a demander's bid for his higher (lower) valued unit is independent of his lower (higher) valuation.

We also introduce some additional notation, the functions $H, T$, and $F$, which are functions of order statistics that help make the statement and proof of theorems 1 and 2 more concise. Let

$$
\begin{aligned}
H\left(z_{1}, z_{2}, G, m, n, l\right)= & \frac{n !}{l ! m !(n-m-l) !}\left(\int_{0}^{z_{1}} \int_{0}^{z_{2}} g\left(v_{1}, v_{2}\right) d v_{2} d v_{1}\right)^{l} \\
& \left(\int_{z_{1}}^{\bar{v}} \int_{0}^{z_{2}} g\left(v_{1}, v_{2}\right) d v_{2} d v_{1}\right)^{m}\left(\int_{z_{1}}^{\bar{v}} \int_{z_{2}}^{\bar{v}} g\left(v_{1}, v_{2}\right) d v_{2} d v_{1}\right)^{n-m-l} .
\end{aligned}
$$

The function $H(\cdot)$ is the probability that in a sample of size $n$ drawn from $g\left(v_{1}, v_{2}\right)$, exactly $l$ observations have the property that $\left(v_{1} \leq z_{1}, v_{2} \leq z_{2}\right)$, exactly $m$ observations have the property that $\left(v_{1}>z_{1}, v_{2} \leq z_{2}\right)$ and exactly $n-m-l$ have the 
property that $\left(v_{1}>z_{1}, v_{2}>z_{2}\right)$. Let

$$
T\left(z_{1}, z_{2}, z_{3}, G, n, k\right)=\frac{\sum_{m, l ; m+2 l=2 n-k+1} H\left(z_{1}, z_{2}, n, m, l\right)}{\sum_{q=2 n-k+2}^{2 n} \sum_{m, l ; m+2 l=q}\left(\frac{\partial H}{\partial z_{1}}+\frac{\partial H}{\partial z_{2}} \frac{1}{z_{3}}\right)} .
$$

The function $T(\cdot)$ describes the amount by which bidders underbid on their lower valued unit in a symmetric Type $M$ equilibrium. Let

$\left(F_{v}^{-i}(x) \mid B\right)=$ Prob (at least $v$ bids made by bidders other than $i$ are less than or equal to $x$ if all bidders except for bidder $i$ use $B$ ).

$\left(F_{v}^{-i}(x) \mid B\right)$ is defined for $v=0, \ldots, 2 n$. For $v>0,\left(F_{v}^{-i}(x) \mid B\right)$ is the cumulative distribution function of the $v$ th order statistic of bids made by $n$ randomly chosen demanders using strategy $B$. Let $f_{v}^{-i}(x) \mid B$ denote the corresponding density function.

Theorem 1. A bidding function is a symmetric undominated type $M$ Bayes-Nash equilibrium only if it equals $\beta\left(v_{1}, v_{2}\right)=\left(\beta_{1}\left(v_{1}, v_{2}\right), \beta_{2}\left(v_{1}, v_{2}\right)\right)$, where:

$$
\beta_{1}\left(v_{1}\right)=v_{1}
$$

and:

$$
\beta_{2}\left(v_{2}\right)= \begin{cases}0 & v_{2} \leq v_{2}^{*} \\ \bar{\beta}_{2}\left(v_{2}\right) & v_{2} \geq v_{2}^{*}\end{cases}
$$

where:

$$
v_{2}^{*}=T\left(0, v_{2}^{*}, \frac{\partial \bar{\beta}_{2}\left(v_{2}^{*}\right)}{\partial v_{2}}, G, n, k\right)
$$

and $\bar{\beta}_{2}$ solves the differential equation:

$$
\bar{\beta}_{2}\left(v_{2}\right)=v_{2}-T\left(\bar{\beta}_{2}\left(v_{2}\right), v_{2}, \frac{\partial \bar{\beta}_{2}\left(v_{2}\right)}{\partial v_{2}}, G, n, k\right),
$$

with the initial conditions:

$$
\begin{aligned}
v_{2}^{*}=0 ; & \text { if } n>k-1 \\
\bar{\beta}_{2}(\bar{v})=\bar{v} ; & \text { if } n<k-1 \\
\bar{\beta}_{2}(0)=0 ; & \text { if } n=k-1 .
\end{aligned}
$$

Theorem 1 is proven using lemmas 1 and 2, which are stated and proven in this subsection and lemmas $3-7$ in the appendix.

Lemma 1. If a bidding function $\beta\left(v_{1}, v_{2}\right)=\left(\beta_{1}\left(v_{1}, v_{2}\right), \beta_{2}\left(v_{1}, v_{2}\right)\right)$, is a symmetric undominated type $M$ Bayes-Nash equilibrium, then $\beta_{1}\left(v_{1}\right)=v_{1}$.

Proof: Suppose all $n+1$ bidders are using the same equilibrium bidding function $B^{*}\left(v_{1}, v_{2}\right)$. For notational ease, let $F_{v}^{-i *}(x)=F_{v}^{-i}(x) \mid B^{*}$. Since $B^{*}$ is symmetric, $\gamma$ is common, and valuations are drawn independently, $F_{v}^{-i *}(x)=F_{v}^{*}(x) ; \forall i$. Bidder $i$ 's expected profit is given in equation (8). 


$$
\begin{aligned}
E \pi^{i}= & \int_{b_{2}^{i}}^{b_{1}^{i}}\left(v_{1}^{i}-M_{2 n-k+1}\right) f_{2 n-k+1}^{*}\left(M_{2 n-k+1}\right) d M_{2 n-k+1} \\
& +\int_{0}^{b_{2}^{i}}\left(v_{1}^{i}+v_{2}^{i}-2 M_{2 n-k+2}\right) f_{2 n-k+2}^{*}\left(M_{2 n-k+2}\right) d M_{2 n-k+2} \\
& +\left(v_{1}^{i}-b_{2}^{i}\right)\left(F_{2 n-k+1}^{*}\left(b_{2}^{i}\right)-F_{2 n-k+2}^{*}\left(b_{2}^{i}\right)\right) .
\end{aligned}
$$

where $M_{v}$ is the $v$ th lowest order statistic of bids made by bidders other than bidder $i$ and $b_{j}^{i}$ is the $j$ th highest bid made by bidder $i$. The first term indicates the profit when the purchase price is between $i$ 's two bids, in which case the purchase price equals $M_{2 n-k+1}$. The second term gives the profit when both of $i$ 's bids are accepted, in which event he obtains two units at a per-unit price of $M_{2 n-k+2}$ and the third term gives the profits when $i$ 's lower bid is the $k+1$ st highest, in which event he receives one unit and pays a per-unit price of $b_{2}^{i}$. In all other cases, $i$ 's profits equal 0.

In equilibrium, bidder $i$ 's two bids, $b_{1}^{i}$ and $b_{2}^{i}$, are chosen to maximize (8) subject to $b_{1}^{i} \geq 0, b_{2}^{i} \geq 0$. The first order necessary (Kuhn-Tucker) conditions are given in equations (9) and (10):

$$
\begin{gathered}
\frac{\partial E \pi^{i}}{\partial b_{1}^{i}}=\left(v_{1}^{i}-b_{1}^{i}\right) f_{2 n-k+1}^{*}\left(b_{1}^{i}\right)=0 ; \quad b_{1}^{i}>0, \\
\leq 0 ; \quad b_{1}^{i}=0, \\
\frac{\partial E \pi^{i}}{\partial b_{2}^{i}}=\left(v_{2}^{i}-b_{2}^{i}\right) f_{2 n-k+2}^{*}\left(b_{2}^{i}\right)-\left(F_{2 n-k+1}^{*}\left(b_{2}^{i}\right)-F_{2 n-k+2}^{*}\left(b_{2}^{i}\right)\right)=0 ; \quad b_{2}^{i}>0, \\
\leq 0 ; \quad b_{2}^{i}=0 .
\end{gathered}
$$

If $f_{2 n-k+1}^{*}\left(b_{1}^{i}\right)>0$, then $B^{*}$ is separable and $B_{1}^{*}\left(v_{1}\right)=v_{1}$ for $v_{1} \geq 0$. If $f_{2 n-k+1}^{*}\left(b_{1}^{i}\right)=0$, then there can be more than one solution but any strategy is weakly dominated by $B_{1}^{*}\left(v_{1}\right)=v_{1} . b_{1}^{i}<v_{1}^{i}$ results is profits lower than $b_{1}^{i}=v_{1}^{i}$ if $b_{1}^{i}<M_{2 n-k+1}<v_{1}^{i}$ and equal otherwise. $b_{1}^{i}>v_{1}^{i}$ results in profits to bidder $i$ lower than $b_{1}^{i}=v_{1}^{i}$ if $v_{1}^{i}<M_{2 n-k+1}<b_{1}^{i}$ and equal otherwise.

We have shown that in equilibrium, each bidder's higher bid equals his higher valuation. We derive the lower bid in lemmas 2-7. Lemma 2 contains the most important property of the lower bid, that it must satisfy the differential equation in (6), which describes the exact amount of strategic underbidding. The proof of lemmas 3-7 establish initial conditions and boundary values for equation (6). We cannot establish one initial condition that always holds. However, given the number of bidders and units sold, we can always derive one initial condition. The differences are illustrated in figure 1.

Figure 1 depicts the general form that $\beta_{2}\left(v_{2}\right)$ may have. There are three possible cases. In the first case, in which the number of bidders if strictly less than the number of units being sold, that is, $n<k-1$, it must be the case that $\beta_{2}(\bar{v})=\bar{v}$. It is possible that $v_{2}^{*}>0$ and $\beta_{2}\left(v_{2}^{\circ}\right)=0$ for all $v_{2}^{\circ} \leq v_{2}^{*}$. In the second case, where $n>k-1, v_{2}^{*}$ must equal 0 . Thus $\beta_{2}\left(v_{2}\right)$ is required to be strictly monotone at all values of $v_{2}$ from 

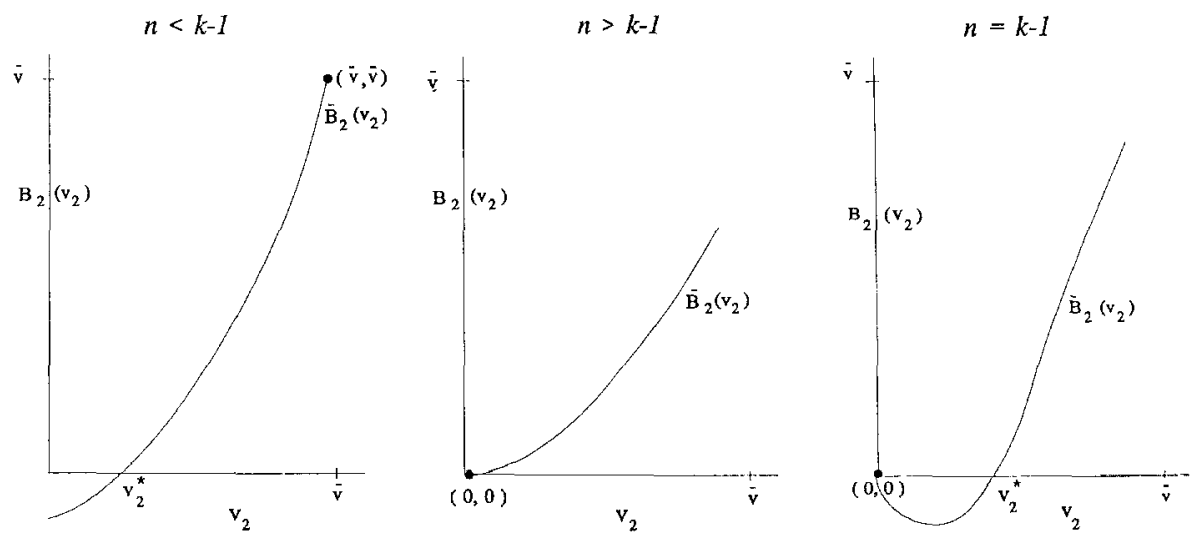

Figure 1. $\beta_{2}\left(v_{2}\right)$ under different values of $n$ and $k$.

0 to $\vec{v}$. In the third case, when $n=k-1$, it must be the case that $\bar{\beta}_{2}(0)=0$. However $v_{2}^{*}$ may be strictly greater than 0 so that for all $v_{2}^{0} \leq v_{2}^{*}, \beta_{2}\left(v_{2}^{\circ}\right)=0$.

Lemma 2. If $B^{*}$ is a Type $M$ undominated symmetric Bayes-Nash equilibrium, then $B_{2}^{*}\left(v_{1}, v_{2}\right)=\beta_{2}\left(v_{2}\right) ;$ where $\beta_{2}\left(v_{2}\right)=\bar{\beta}_{2}\left(v_{2}\right) ;$ for $v_{2} \geq v_{2}^{*}$ and $\bar{\beta}_{2}\left(v_{2}\right)$ solves:

$$
\bar{\beta}_{2}\left(v_{2}\right)=v_{2}-T\left(\bar{\beta}_{2}\left(v_{2}\right), v_{2}, \frac{\partial \bar{\beta}_{2}\left(v_{2}\right)}{\partial v_{2}}, G, n, k\right) .
$$

Proof: The first order conditions require $b_{2}^{i}=v_{2}^{i}-\left(F_{2 n-k+1}^{*}\left(b_{2}^{i}\right)-F_{2 n-k+2}^{*}\left(b_{2}^{i}\right)\right) /$ $f_{2 n-k+2}^{*}\left(b_{2}^{i}\right)$ for any symmetric equilibrium bidding function $B^{*}$. By lemma 10 , $f_{2 n-k+2}^{*}\left(b_{2}^{i}\right)>0$ so the last expression is defined. Since $B^{*}$ is being used by all players and $B_{1}^{*}\left(v_{1}\right)=v_{1}$ :

$$
\operatorname{Prob}\left(v_{1} \leq B_{1}^{-1 *}\left(b_{2}^{i}\right)\right)=\operatorname{Prob}\left(v_{1} \leq b_{2}^{i}\right) .
$$

Also, because $\frac{\partial B_{2}^{*}\left(v_{2}\right)}{\partial v_{2}}>0$ for $v_{2}>\hat{v}_{2}$ and $B_{2}^{*}\left(v_{2}\right)=0$ for $v_{2} \leq \hat{v}_{2}$ :

$$
\operatorname{Prob}\left(B_{2}^{*}\left(v_{2}\right) \leq b_{2}^{i}\right)=\operatorname{Prob}\left(v_{2} \leq V_{2}\left(b_{2}^{i}\right)\right),
$$

where the function $V_{2}(x):\left[0, B_{2}^{*}(\bar{v})\right] \rightarrow\left[\hat{v}_{2}, \bar{v}\right]$, and $V_{2}(x)=B_{2}^{-1 *}(x)$. Consider the probability that a randomly drawn bidder, named $y \neq i$, submits 2 bids that are less than or equal to $b_{2}^{i}$. The probability that two of $y$ 's bids are less than $b_{2}^{i}$ is the probability of the following event:

$$
\operatorname{Prob}\left(b_{1}^{y} \leq b_{2}^{i}, b_{2}^{y} \leq b_{2}^{i}\right)=\int_{0}^{V_{2}\left(b_{2}^{i}\right)} \int_{0}^{b_{2}^{i}} g\left(v_{1}, v_{2}\right) d v_{1} d v_{2} .
$$

Similarly, exactly one of $y$ 's bids is less than or equal to $b_{2}^{i}$ when either of the two following events occurs:

$$
\operatorname{Prob}\left(b_{1}^{y}>b_{2}^{i}, b_{2}^{y} \leq b_{2}^{i}\right)=\int_{0}^{V_{2}\left(b_{2}^{i}\right)} \int_{b_{2}^{i}}^{\bar{v}} g\left(v_{1}, v_{2}\right) d v_{1} d v_{2},
$$


or

$$
\operatorname{Prob}\left(b_{1}^{y} \leq b_{2}^{i}, b_{2}^{y}>b_{2}^{i}\right)=\int_{V_{2}\left(b_{2}^{i}\right)}^{\bar{v}} \int_{0}^{b_{2}^{i}} g\left(v_{1}, v_{2}\right) d v_{1} d v_{2} .
$$

The last expression equals 0 because it requires that $\left(v_{1}^{y} \leq b_{2}^{i}, v_{2}^{y} \geq V_{2}\left(b_{2}^{i}\right)\right)$, an event that occurs with probability zero; since $\left(F_{2 n-k+1}^{*}\left(b_{2}^{i}\right)-F_{2 n-k+2}^{*}\left(b_{2}^{i}\right)\right)$ / $f_{2 n-k+2}^{*}\left(b_{2}^{i}\right) \geq 0$ (because the numerator is a probability and the denominator is a density), it must be the case that $V_{2}\left(b_{2}^{i}\right) \geq b_{2}^{i}$; but by assumption $v_{1}^{y} \geq v_{2}^{y}$. The probability that demander $y$ makes 0 bids less than or equal to $b_{2}^{i}$ is given by:

$$
\operatorname{Prob}\left(b_{1}^{y}>b_{2}^{i}, b_{2}^{y}>b_{2}^{i}\right)=\int_{V_{2}\left(b_{2}^{i}\right)}^{\bar{v}} \int_{b_{2}^{i}}^{\bar{v}} g\left(v_{1}, v_{2}\right) d v_{1} d v_{2} .
$$

Suppose now that each of the $n$ bidders other than bidder $i$ draws one pair of valuations from $G\left(v_{1}, v_{2}\right)$. Exactly $l$ of the buyers make two bids less than or equal to $b_{2}^{i}$, exactly $m$ buyers make one, and exactly $(n-m-l)$ bidders make zero bids less than or equal to $b_{2}^{i}$. The probability of this event is given by:

Prob (exactly $l$ observations of $B_{1}^{*}\left(v_{1}^{-i}\right) \leq b_{2}^{i}$, exactly $m+l$ observations of $\left.B_{2}^{*}\left(v_{2}^{-i}\right) \leq b_{2}^{i}\right)$

$$
\begin{aligned}
= & \frac{n !}{l ! m !(n-m-l) !}\left(\int_{0}^{V_{2}\left(b_{2}^{i}\right)} \int_{0}^{b_{2}^{i}} g\left(v_{1}, v_{2}\right) d v_{1} d v_{2}\right)^{l} \\
& \left(\int_{0}^{V_{2}\left(b_{2}^{i}\right)} \int_{b_{2}^{i}}^{\bar{v}} g\left(v_{1}, v_{2}\right) d v_{1} d v_{2}\right)^{m}\left(\int_{V_{2}\left(b_{2}^{i}\right)}^{\bar{v}} \int_{b_{2}^{i}}^{\bar{v}} g\left(v_{1}, v_{2}\right) d v_{1} d v_{2}\right)^{n-m-l} .
\end{aligned}
$$

The previous expression equals $H\left(b_{2}^{i}, V_{2}\left(b_{2}^{i}\right), G, n, m, l\right)$ where $H$ is as defined in equation (1). It follows that:

$$
\begin{aligned}
\left(F_{2 n-k+1}^{*}\left(b_{2}^{i}\right)-F_{2 n-k+2}^{*}\left(b_{2}^{i}\right)\right) & =\sum_{l, m ; 2 l+m=2 n-k+1} H\left(b_{2}^{i}, V_{2}\left(b_{2}^{i}\right), G, n, m, l\right), \\
\left.F_{2 n-k+2}^{*}\left(b_{2}^{i}\right)\right) & =\sum_{q=2 n-k+2} \sum_{l, m ; 2 l+m=q} H\left(b_{2}^{i}, V_{2}\left(b_{2}^{i}\right), G, n, m, l\right),
\end{aligned}
$$

and

$$
\left.f_{2 n-k+2}^{*}\left(b_{2}^{i}\right)\right)=\sum_{q=2 n-k+2}^{2 n} \sum_{l, m: 2 l+m=q}\left(\frac{\partial H}{\partial b_{2}^{i}}+\frac{\partial H}{\partial V_{2}} \frac{\partial V_{2}}{\partial b_{2}^{i}}\right) .
$$

The last three equations imply that:

$$
\left(F_{2 n-k+1}^{*}\left(b_{2}^{i}\right)-F_{2 n-k+2}^{*}\left(b_{2}^{i}\right)\right) / f_{2 n-k+2}^{*}\left(b_{2}^{i}\right)=T\left(b_{2}^{i}, V_{2}\left(b_{2}^{i}\right),\left(\frac{\partial V_{2}}{\partial b_{2}^{i}}\right)^{-1}, G, n, k\right) .
$$

Since all bidders are using the same strategy, $b_{2}^{i}$ must equal $B_{2}^{*}\left(v_{2}^{i}\right)$. Therefore:

$$
T\left(b_{2}^{i}, V_{2}\left(b_{2}^{i}\right),\left(\frac{\partial V_{2}}{\partial b_{2}^{i}}\right)^{-1}, G, n, k\right)=T\left(B_{2}^{*}\left(v_{2}^{i}\right), v_{2}^{i}, \frac{\partial B_{2}^{*}\left(v_{2}\right)}{\partial v_{2}}, G, n, k\right) .
$$


Using equations (10), (21) and (22), we see that equation (6) and the second part of equation (4) must hold.

Proof of Theorem 1. The proof follows directly from lemmas $1-7$. It has now been shown that $B^{*}\left(v_{1}, v_{2}\right)$ is a type $\mathrm{M}$ undominated symmetric Bayes-Nash equilibrium, only if it equals $\beta$.

There is underrevelation on the lower-valued unit for the following reason: since there is positive probability that a demander's lower bid is the $k+1$ st highest, he has some incentive to underbid for it in order to lower the price he pays for the unit he receives (the fact that the lower bid is the $k+1$ st highest implies that the higher bid is among the $k$ highest, and therefore the demander receives exactly one unit). There is no incentive to underbid on the higher-valued unit, since in the event that the demander's higher bid is the $k+1$ st highest, he wins no units, and his profits are zero. Overbidding is always a dominated strategy.

The two symmetric equilibrium bids are separable, indicating that the extent of underrevelation on the lower unit depends only upon the rank of the unit, the distribution of valuations, the number of bidders and the number of units sold, and is independent of the bidder's higher valuation and his higher bid. The independence results from the fact that the price paid is independent of the amount of the higher bid, and therefore the gains from lowering the final price depend only upon how many bids are accepted in the event that the lower bid is the $k+1$ st highest.

\subsection{A simple example}

Suppose $\gamma(v)$ is uniform on the interval from 0 to $1, n+1=2$ and $k=3$. We know that $\beta_{1}\left(v_{1}^{i}\right)=v_{1}^{i}$ in an undominated symmetric equilibrium. The calculation of $\beta_{2}\left(v_{2}^{i}\right)$, which equals $v_{2}^{i}-T(\cdot)$, proceeds in the following manner. First note that $\frac{n !}{l ! m !(n-m-l) !}=1$. Using the fact that $v_{1} \geq v_{2}$, we can derive the following equation which gives the probability that a bidder makes exactly two bids less than or equal to $b_{2}^{i}$ :

$$
\int_{0}^{V_{2}\left(b_{2}^{i}\right)} \int_{0}^{b_{2}^{i}} g\left(v_{1}, v_{2}\right) d v_{1} d v_{2}=\int_{0}^{b_{2}^{i}} \int_{0}^{b_{2}^{i}} g\left(v_{1}, v_{2}\right) d v_{1} d v_{2}=\left(b_{2}^{i}\right)^{2} .
$$

The probability that a randomly chosen bidder makes exactly one bid less than or equal to $b_{2}^{i}$ equals:

$$
\int_{0}^{V_{2}\left(b_{2}^{i}\right)} \int_{b_{2}^{i}}^{\bar{v}} g\left(v_{1}, v_{2}\right) d v_{1} d v_{2}=2 V_{2}\left(b_{2}^{i}\right)-\left(V_{2}\left(b_{2}^{i}\right)\right)^{2}-\left(b_{2}^{i}\right)^{2}
$$

and again using $v_{1} \geq v_{2}$, we can derive the probability that a randomly chosen bidder makes exactly zero bids that are less than or equal to $b_{2}^{i}$. The probability is given by:

$$
\int_{V_{2}\left(b_{2}^{i}\right)}^{\bar{v}} \int_{b_{2}^{i}}^{\bar{v}} g\left(v_{1}, v_{2}\right) d v_{1} d v_{2}=\int_{V_{2}\left(b_{2}^{i}\right)}^{\bar{v}} \int_{V_{2}\left(b_{2}^{i}\right)}^{\bar{v}} g\left(v_{1}, v_{2}\right) d v_{1} d v_{2}=\left(1-V_{2}\left(b_{2}^{i}\right)\right)^{2} .
$$


The numerator of $T$ equals the following expression (note $2 n-k+1=0$ ):

$$
\sum_{(m, l ; m+2 l=2 n-k+1)} H\left(b_{2}^{i}, V_{2}\left(b_{2}^{i}\right), G\left(v_{1}, v_{2}\right), n, m, l\right)=\left(1-V_{2}\left(b_{2}^{i}\right)\right)^{2} .
$$

Next, we derive the denominator of $T . F_{2 n-k+2}\left(b_{2}^{i}\right)$ equals $\left(b_{2}^{i}\right)^{2}+2 V_{2}\left(b_{2}^{i}\right)-$ $\left(V_{2}\left(b_{2}^{i}\right)\right)^{2}-\left(b_{2}^{i}\right)^{2}$ and therefore $f_{2 n-k+2}\left(b_{2}^{i}\right)=\left(2-2 V_{2}\left(b_{2}^{i}\right)\right) V_{2}^{\prime}\left(b_{2}^{i}\right)$. Since the equilibrium is symmetric, $V_{2}\left(b_{2}^{i}\right)=v_{2}^{i}$. The solutions to the first order necessary conditions are given by:

$$
\begin{gathered}
b_{1}^{i}=\beta_{1}\left(v_{1}\right)=v_{1}^{i} ; \quad b_{1}^{i}>0, \\
\geq v_{1}^{i} ; \quad b_{1}^{i}=0, \\
b_{2}^{i}=\beta_{2}\left(v_{2}\right)=v_{2}^{i}-\frac{\left(1-v_{2}^{i}\right)^{2}}{\left(2-2 v_{2}^{i}\right) V_{2}^{\prime}\left(b_{2}^{i}\right)} ; \quad b_{2}^{i}>0, \\
\geq v_{2}^{i}-\frac{\left(1-v_{2}^{i}\right)^{2}}{\left(2-2 v_{2}^{i}\right) V_{2}^{\prime}\left(b_{2}^{i}\right)} ; \quad b_{2}^{i}=0,
\end{gathered}
$$

with the initial condition $\beta_{2}(1)=1$ because $n<k-1$. Solving for $V_{2}^{\prime}\left(b_{2}^{i}\right)$ we obtain:

$$
V_{2}^{\prime}\left(b_{2}^{i}\right)=\frac{1-v_{2}^{i}}{2\left(v_{2}^{i}-b_{2}^{i}\right)} .
$$

A solution can be found by setting $b_{2}^{i}=\left(v_{2}^{i}\right)^{2}$ which implies that $V_{2}\left(b_{2}^{i}\right)=\left(b_{2}^{i}\right)^{1 / 2}$ and also that $V_{2}^{\prime}=\frac{1}{2}\left(v_{2}^{i}\right)^{-1}$. We obtain:

$$
\beta\left(v_{1}, v_{2}\right)=\left(v_{1},\left(v_{2}\right)^{2}\right)
$$

\subsection{Sufficient conditions}

In theorem 1 we provided necessary conditions for a bidding function to be a symmetric undominated Type $M$ Bayes-Nash equilibrium. In theorem 2 sufficient conditions are given for $\beta$ to be an equilibrium. There are two conditions: $\mathrm{A}$ and $\mathrm{B}$. Condition A insures that the appropriate second order conditions are satisfied; if all other demanders use $\beta$, the payoff function of bidder $i$ is concave in bidder $i$ 's strategy. Condition $\mathbf{B}$ insures that $\beta$ is type $\mathbf{M}$.

Condition A: $\beta$ satisfies Condition $\mathbf{A}$ if:

$$
\begin{gathered}
\sum_{q=2 n-k+2}^{2 n} \sum_{m, l ; 2 l+m=q}\left(\frac{\partial H}{\partial W_{2}} \frac{\partial^{2} W_{2}}{\partial b_{2}^{2}}+\frac{\partial^{2} H}{\partial b_{2}^{2}}+2 \frac{\partial H}{\partial b_{2}} \frac{\partial H}{\partial W_{2}} \frac{\partial W_{2}}{\partial b_{2}}+\frac{\partial^{2} H}{\partial W_{2}^{2}}\left(\frac{\partial W_{2}}{\partial b_{2}}\right)^{2}\right) \\
*\left(W_{2}\left(b_{2}\right)-b_{2}\right)<\sum_{q=2 n-k+1 m, l ; 2 l+m=q}^{2 n}\left(\frac{\partial H}{\partial b_{2}}+\frac{\partial H}{\partial W_{2}} \frac{\partial W_{2}}{\partial b_{2}}\right)
\end{gathered}
$$

for all $b_{2}$ such that $0 \leq b_{2} \leq \bar{v}$ where $W_{2}(x):\left[0, \beta_{2}(\bar{v})\right] \rightarrow\left[v_{2}^{*}, \bar{v}\right], W_{2}(x)=\beta_{2}^{-1}(x)$, where $H=H\left(b_{2}, W_{2}\left(b_{2}\right), G, n, m, l\right)$

Condition B: $\beta$ satisfies condition B if:

$$
\frac{1-\partial T / \partial v_{2}}{1+\partial T / \partial \beta_{2}}>0 ; \quad \beta_{2}>0
$$


Theorem 2. Suppose that $\beta$ satisfies (3)-(7) and conditions $A$ and $B$. Under conditions $A$ and $B$, the bidding function $\beta\left(v_{1}, v_{2}\right)=\left(\beta_{1}\left(v_{1}, v_{2}\right), \beta_{2}\left(v_{1}, v_{2}\right)\right)$ is a symmetric undominated Type $M$ Bayes-Nash equilibrium.

Proof: The theorem is proven in lemmas 8 and 9 in the appendix.

It can be readily verified that the second order conditions hold for the example in the last subsection.

\section{Summary and concluding remarks}

We generalized some important theoretical properties of a uniform price sealed bid auction with "first rejected bid" pricing to an independent private values environment with two-unit demands. We considered a class of bidding functions called type $\mathrm{M}$, essentially a general type of continuous monotonicity. A necessary condition for a bidding function to be a type $M$ symmetric undominated Bayes-Nash equilibrium was derived. The dominant strategy equilibrium of the single-unit demand environment results as a special case. In any equilibrium, there is underbidding for each demander's lower-valued unit, as demanders, even as they behave noncooperatively, underreveal demand in an attempt to shift the market price in their favor. An interesting property of type $\mathrm{M}$ equilibria, separability, is also obtained. A sufficient condition for a solution to the necessary conditions to be an equilibrium is also deduced and an example of a type $\mathbf{M}$ equilibrium is provided.

Clearly, intuition which follows only from knowledge of equilibrium properties of uniform price sealed bid auctions in the single-unit demand environment is not valid when considering multi-unit demand environments. Although this has been known for some time, this paper extends previous results by characterizing the precise extent of strategic behavior in a simple uniform price auction in a multi-unit demand environment.

\section{A. Additional proofs}

Lemma 3. $\beta_{2}\left(v_{2}^{*}\right)=0 ; \quad v_{2}^{*}=T\left(0, v_{2}^{*}, \frac{\partial \bar{\beta}_{2}\left(v_{2}^{*}\right)}{\partial v_{2}}, G, n, k\right)$.

Proof: Equation (10) implies that for $b_{2}^{i}=0,0 \geq V_{2}(0)-T\left(0, V_{2}(0),\left(\frac{\partial V_{2}(0)}{\partial b_{2}^{i}}\right)^{-1}\right.$, $G, n, k)$. The inequality holds with equality if $V_{2}(0)=T\left(0, V_{2}(0),\left(\frac{\partial V_{2}(0)}{\partial b_{2}^{i}}\right)^{-1}, G, n, k\right)$,
which implies that $V_{2}(0)=v_{2}^{*}$.

Lemma 4. $\beta_{2}\left(v_{2}\right)=0$; if $v_{2} \leq v_{2}^{*}$. Proof: Consider any $v_{2}^{\circ}<v_{2}^{*}$. Then $0>v_{2}^{\circ}-T\left(0, V_{2}(0),\left(\frac{\partial V_{2}(0)}{\partial b_{2}^{i}}\right)^{-1}, G, n, k\right)$. By
equation $(10), B_{2}^{*}\left(v_{2}^{\circ}\right)=0$. $\square$

Lemma 5. $v_{2}^{*}=0$; if $n>k-1$. 
Proof: Consider $v_{2}^{*}=T\left(0, v_{2}^{*}, \frac{\partial B_{2}^{*}\left(v_{2}^{*}\right)}{\partial v_{2}}, G, n, k\right)$. By lemma 10 , the denominator of the last equation, $\int_{2 n-k+2}^{*}(0)>0$. If the numerator is equal to zero, it would imply that $v_{2}^{*}=0$. The numerator is:

$$
\begin{aligned}
& \quad \sum_{l, m ; 2 l+m=2 n-k+1} H\left(0, V_{2}(0), G, n, m, l\right)=\sum_{l, m ; 2 l+m=2 n-k+1} \frac{n !}{l ! m !(n-m-l) !} \\
& *\left(\int_{0}^{0} \int_{0}^{V_{2}(0)} g\left(v_{1}, v_{2}\right) d v_{2} d v_{1}\right)^{l}\left(\int_{0}^{\bar{v}} \int_{0}^{V_{2}(0)} g\left(v_{1}, v_{2}\right) d v_{2} d v_{1}\right)^{m} \\
& *\left(\int_{0}^{\bar{v}} \int_{V_{2}(0)}^{\bar{v}} g\left(v_{1}, v_{2}\right) d v_{2} d v_{1}\right)^{n-m-l} .
\end{aligned}
$$

The last expression equals 0 unless $l=0$. If $l$ equals 0 , the expression equals:

$$
\sum_{m=2 n-k+1} \frac{n !}{m !(n-m) !}\left(\int_{0}^{\bar{v}} \int_{0}^{V_{2}(0)} g(\cdot) d v_{2} d v_{1}\right)^{m}\left(\int_{0}^{\bar{v}} \int_{V_{2}(0)}^{\bar{v}} g(\cdot) d v_{2} d v_{1}\right)^{n-m}
$$

Since $m \geq 0$, the last expression equals 0 if $n>k-1$. Therefore, $v_{2}^{*}=0$ when $n>k-1$.

Lemma 6. $\bar{\beta}_{2}(\bar{v})=\bar{v} ;$ if $n<k-1$.

Proof: Consider $T\left(B_{2}^{*}(\bar{v}), \bar{v}, \frac{\partial B_{2}^{*}(\bar{v})}{\partial v_{2}}, G, n, k\right)$. The denominator of $T$ is positive by lemma 10. If $v_{2}^{i}=\bar{v}$, the numerator of $T$ equals:

$$
\begin{aligned}
& \quad \sum_{l, m ; 2 l+m=2 n-k+1} H\left(B_{2}^{*}(\bar{v}), \bar{v}, G, n, m, l\right)=\sum_{l, m ; 2 l+m=2 n-k+1} \frac{n !}{l ! m !(n-m-l) !} \\
& *\left(\int_{0}^{B_{2}^{*}(\bar{v})} \int_{0}^{\bar{v}} g\left(v_{1}, v_{2}\right) d v_{2} d v_{1}\right)^{l}\left(\int_{B_{2}^{*}(\bar{v})}^{\bar{v}} \int_{0}^{\bar{v}} g\left(v_{1}, v_{2}\right) d v_{2} d v_{1}\right)^{m} \\
& *\left(\int_{B_{2}^{*}(\bar{v})}^{\bar{v}} \int_{\bar{v}}^{\bar{v}} g\left(v_{1}, v_{2}\right) d v_{2} d v_{1}\right)^{n-m-l} .
\end{aligned}
$$

The previous equation equals 0 unless $n=m+l$. It also equals 0 unless $2 l+m=$ $2 n-k+1 . n=m+l$ and $2 l+m=2 n-k+1$ cannot be satisfied simultaneously if $n<k-1$ since $(2 l+m=2 n-k+1) \Leftrightarrow(2 l+n-l=2 n-k+1) \Leftrightarrow(l=n-k+1)$. Since $l \geq 0$, it follows that if $n<k-1, T\left(B_{2}^{*}(\bar{v}), \bar{v}, \frac{\partial B_{2}^{*}(\bar{v})}{\partial v_{2}}, G, n, k\right)=0$ implying that

Lemma 7. $\bar{\beta}_{2}(0)=0$; if $n=k-1$.

Proof: Define $\bar{B}_{2}^{*}(0)$ to be: $\bar{B}_{2}^{*}(0)=0-T\left(0, \bar{B}_{2}^{*}(0), \frac{\partial \bar{B}_{2}^{*}(0)}{\partial v_{2}}, G, n, k\right)$. The numerator 
of Tequals:

$$
\begin{aligned}
& \quad \sum_{l, m ; 2 l+m=2 n-k+1} H\left(\bar{B}_{2}^{*}(0), 0, G, n, m, l\right)=\sum_{l, m ; 2 l+m=2 n-k+1} \frac{n !}{l ! m !(n-m-l) !} \\
& *\left(\int_{0}^{\bar{B}_{2}^{*}(0)} \int_{0}^{o} g\left(v_{1}, v_{2}\right) d v_{2} d v_{1}\right)^{l}\left(\int_{\bar{B}_{2}^{*}(0)}^{\bar{v}} \int_{0}^{o} g\left(v_{1}, v_{2}\right) d v_{2} d v_{1}\right)^{m} \\
& *\left(\int_{\bar{B}_{2}^{*}(0)}^{\bar{v}} \int_{0}^{\bar{v}} g\left(v_{1}, v_{2}\right) d v_{2} d v_{1}\right)^{n-m-l} .
\end{aligned}
$$

Equation (36) equals zero unless $l=0$ and $m=0$. If $l=m=0,(36)$ equals zero unless $2 n-k+1$ equals zero. If $2 n-k+1=0$, then $n \neq k-1$ if $n>0$. Therefore, for $n=k-1, T\left(0, \bar{B}_{2}^{*}(0), \frac{\partial \bar{B}_{2}^{*}(0)}{\partial v_{2}}, G, n, k\right)=0$, which implies that $\bar{B}_{2}^{*}(0)=0$ when $n=$
$k-1$

Lemma 8. If condition $B$ holds, $\beta$ is Type $M$.

Proof: Suppose condition B holds. Clearly $\beta(0,0)=(0,0)$ and $\beta$ is continuous in $v_{1}$ and $v_{2} \cdot \hat{v}_{1}\left(v_{2}\right)=0, \hat{v}_{2}\left(v_{1}\right)=v_{2}^{*}$ and $\beta_{1}(\bar{v}, \bar{v})=\bar{v} \geq \beta_{2}(\bar{v}, \bar{v})$. Since $\beta_{1}\left(v_{1}\right)=v_{1}, \frac{\partial \beta_{1}}{\partial v_{1}}>0$. Now consider $\beta_{2}\left(v_{2}\right)=v_{2}-T\left(\beta_{2}, v_{2}, \frac{\partial \beta_{2}}{\partial v_{2}}, G, n, k\right)$ for $\beta_{2}>0$. The partial derivative of $\beta_{2}$ with respect to $v_{2}$ satisfies:

$$
\frac{\partial \beta_{2}}{\partial v_{2}}=1-\frac{\partial T}{\partial \beta_{2}} \frac{\partial \beta_{2}}{\partial v_{2}}-\frac{\partial T}{\partial v_{2}}=\frac{1-\partial T / \partial v_{2}}{1+\partial T / \partial \beta_{2}}>0
$$

Finally, since $\beta$ is separable: $\frac{\partial \beta_{1}}{\partial v_{2}}=\frac{\partial \beta_{2}}{\partial v_{1}}=0$.

Lemma 9. If conditions $A$ and $B$ hold, $\beta$ is an undominated Type $M$ symmetric Bayes-Nash equilibrium.

Suppose all bidders except for bidder $i$ are using the bidding function $\beta\left(v_{1}, v_{2}\right)$. The objective function for bidder $i$ is given by:

$$
\begin{aligned}
E \pi^{i} \mid \beta= & \int_{b_{2}^{i}}^{b_{1}^{i}}\left(v_{1}^{i}-M_{2 n-k+1}\right) f_{2 n-k+1}\left(M_{2 n-k+1}\right) d M_{2 n-k+1} \\
& +\int_{0}^{b_{2}^{i}}\left(v_{1}^{i}+v_{2}^{i}-2 M_{2 n-k+2}\right) f_{2 n-k+2}\left(M_{2 n-k+2}\right) d M_{2 n-k+2} \\
& +\left(v_{1}^{i}-b_{2}^{i}\right)\left(F_{2 n-k+1}\left(b_{2}^{i}\right)-F_{2 n-k+2}\left(b_{2}^{i}\right)\right)
\end{aligned}
$$

where $F_{v}(x)=F_{v}^{-i} \mid \beta$. Bidder $i$ chooses $b_{1}^{i}$ and $b_{2}^{i}$, to maximize the objective function subject to $b_{1}^{i} \geq 0, b_{2}^{i} \geq 0$. By lemma 11 , the objective function is twice differentiable. 
The first order necessary conditions are given by equations (39) and (40):

$$
\begin{aligned}
& \frac{\partial E \pi^{i}}{\partial b_{1}^{i}}=\left(v_{1}^{i}-b_{1}^{i}\right) f_{2 n-k+1}\left(b_{1}^{i}\right)=0 ; \quad b_{1}^{i}>0, \\
& \leq 0 ; \quad b_{1}^{i}=0, \\
& \frac{\partial E \pi^{i}}{\partial b_{2}^{i}}=\left(v_{2}^{i}-b_{2}^{i}\right) f_{2 n-k+2}\left(b_{2}^{i}\right)-\left(F_{2 n-k+1}\left(b_{2}^{i}\right)-F_{2 n-k+2}\left(b_{2}^{i}\right)\right)=0 ; \quad b_{2}^{i}>0, \\
& \leq 0 ; \quad b_{2}^{i}=0 .
\end{aligned}
$$

The second derivatives are (omitting the superscript designating demander for notational ease):

$$
\begin{aligned}
\frac{\partial^{2} E \pi}{\partial b_{1}^{2}} & =\left(v_{1}-b_{1}\right) f_{2 n-k+1}^{\prime}\left(b_{1}\right)-f_{2 n-k+1}\left(b_{1}\right), \\
\frac{\partial^{2} E \pi}{\partial b_{1} \partial b_{2}} & =\frac{\partial^{2} E \pi}{\partial b_{2} \partial b_{1}}=0, \\
\frac{\partial^{2} E \pi}{\partial b_{2}^{2}} & =\left(v_{2}-b_{2}\right) f_{2 n-k+2}^{\prime}\left(b_{2}\right)-f_{2 n-k+1}\left(b_{2}\right) .
\end{aligned}
$$

The second order conditions are then: $\frac{\partial^{2} E \pi}{\partial b_{1}^{2}}<0$, and $\frac{\partial^{2} E \pi}{\partial b_{2}^{2}}<0$.

It follows from (39) that if $f_{2 n-k+1}\left(b_{2}^{i}\right)>0$, then $b_{1}=v_{1}$ for $v_{1} \geq 0$. If $f_{2 n-k+1}\left(b_{2}^{i}\right)=0$, then there can be more than one solution to (39) but any strategy is weakly dominated by $b_{1}=v_{1}$. Therefore $b_{1}^{i}=\beta_{1}\left(v_{1}^{i}\right)=v_{1}^{i}$. Since $\beta$ being used by all players besides $i$ and $\beta$ is type $\mathrm{M}$, we can derive the following equation: Prob (exactly $l$ observations of $\beta_{1}\left(v_{1}^{-i}\right) \leq b_{2}^{i}$, exactly $m+$ lobservations of $\left.\beta_{2}\left(v_{2}^{-i}\right) \leq b_{2}^{i}\right)$

$$
\begin{aligned}
= & \frac{n !}{l ! m !(n-m-l) !}\left(\int_{0}^{W_{2}\left(b_{2}^{i}\right)} \int_{0}^{b_{2}^{i}} g\left(v_{1}, v_{2}\right) d v_{1} d v_{2}\right)^{l} \\
& \left(\int_{0}^{W_{2}\left(b_{2}^{i}\right)} \int_{b_{2}^{i}}^{\tilde{j}} g\left(v_{1}, v_{2}\right) d v_{1} d v_{2}\right)^{m}\left(\int_{W_{2}\left(b_{2}^{i}\right)}^{\bar{v}} \int_{b_{2}^{i}}^{\bar{v}} g\left(v_{1}, v_{2}\right) d v_{1} d v_{2}\right)^{n-m-l} .
\end{aligned}
$$

The previous expression equals $H\left(b_{2}^{i}, W_{2}\left(b_{2}^{i}\right), G, n, m, l\right)$, and it follows that:

$$
\left(F_{2 n-k+1}\left(b_{2}^{i}\right)-F_{2 n-k+2}\left(b_{2}^{i}\right)\right) / f_{2 n-k+2}\left(b_{2}^{i}\right)=T\left(b_{2}^{i}, W_{2}\left(b_{2}^{i}\right),\left(\frac{\partial W_{2}\left(b_{2}^{i}\right)}{\partial b_{2}^{i}}\right)^{-1}, G, n, k\right) \text {. }
$$

We can rewrite the first order condition in $(40)$ for $b_{2}^{i}>0$ as:

$$
b_{2}^{i}=v_{2}^{i}-T\left(b_{2}^{i}, W_{2}\left(b_{2}^{i}\right),\left(\frac{\partial W_{2}\left(b_{2}^{i}\right)}{\partial b_{2}^{i}}\right)^{-1}, G, n, k\right) .
$$

One solution to the last equation is to set $b_{2}^{i}=\beta_{2}\left(v_{2}^{i}\right)$ :

$$
\beta_{2}\left(v_{2}^{i}\right)=v_{2}^{i}-T\left(\beta_{2}\left(v_{2}^{i}\right), W_{2}\left(\beta_{2}\left(v_{2}^{i}\right)\right),\left(\frac{\partial W_{2}}{\partial \beta_{2}}\right)^{-1}, G, n, k\right) .
$$


Recalling the fact that $W(x)=\beta_{2}^{-1}(x)$, we see that:

$$
\beta_{2}\left(v_{2}^{i}\right)=v_{2}^{i}-T\left(\beta_{2}\left(v_{2}^{i}\right), v_{2}^{i}, \frac{\partial \beta_{2}\left(v_{2}^{i}\right)}{\partial v_{2}}, G, n, k\right)
$$

The first order conditions also imply that for $b_{2}^{i}=0$ :

$$
0 \geq W_{2}(0)-T\left(\beta_{2}\left(v_{2}^{i}\right), v_{2}^{i}, \frac{\partial \beta_{2}}{\partial v_{2}}, G, n, k\right) .
$$

The last inequality holds with equality when $W_{2}(0)=v_{2}^{*}$. Now consider some $v_{2}^{\circ}<v_{2}^{*}$.

$$
0>v_{2}^{\circ}-T\left(0, v_{2}^{*},\left(\frac{\partial W_{2}(0)}{\partial b_{2}^{i}}\right)^{-1}, G, n, k\right)
$$

$\operatorname{By}(40), b_{2}\left(v_{2}^{\circ}\right)=\beta_{2}\left(v_{2}^{\circ}\right)=0$.

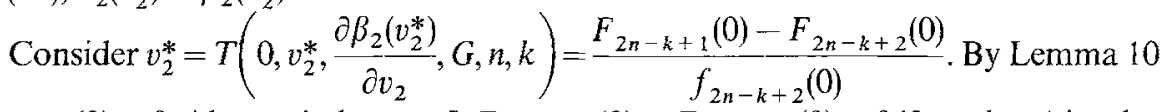
$f_{2 n-k+2}(0)>0$. Also, as in lemma $5, F_{2 n-k+1}(0)-F_{2 n-k+2}(0)=0$ if $n>k-1$ implying that $v_{2}^{*}=0$ if $n>k-1$. As in lemma $6, T\left(\beta_{2}(\bar{v}), \bar{v}, \frac{\partial \beta_{2}(\bar{v})}{\partial v_{2}}, G, n, k\right)=0$ if $n<k-1$, and therefore $\beta_{2}(\bar{v})=\bar{v}$ if $n<k-1$. Finally, as in lemma $7, T\left(\bar{\beta}_{2}(0), 0, \frac{\partial \bar{\beta}_{2}(0)}{\partial v_{2}}, G, n, k\right)=$ 0 , implying that $\bar{\beta}(0)=0$, for $n=k-1$.

We have now shown that $\beta\left(v_{1}, v_{2}\right)$ is a solution to the first order conditions in (39) and (40). If the appropriate second order conditions hold, $\beta$ is a best response. The second order conditions are:

$$
\begin{aligned}
& \frac{\partial^{2} E \pi}{\partial b_{1}^{2}}=\left(v_{1}-b_{1}\right) f_{2 n-k+1}^{\prime}\left(b_{1}\right)-f_{2 n-k+1}\left(b_{1}\right)<0 \\
& \frac{\partial^{2} E \pi}{\partial b_{2}^{2}}=\left(v_{2}-b_{2}\right) f_{2 n-k+2}^{\prime}\left(b_{2}\right)-f_{2 n-k+1}\left(b_{2}\right)<0 .
\end{aligned}
$$

$\frac{\partial^{2} E \pi}{\partial b_{1}^{2}}<0$ is satisfied if $b_{1}=v_{1}$ and $f_{2 n-k+1}\left(b_{1}\right)>0$. If $f_{2 n-k+1}\left(b_{1}\right)=0$, then many $\partial b_{1}^{2}$
solutions are possible but $b_{1}=v_{1}$ dominates any solution that has $b_{1} \neq v_{1} \cdot \frac{\partial^{2} E \pi}{\partial b_{2}^{2}}<0$
is insured by condition $\mathrm{A}$. To see this, consider:

$$
f_{2 n-k+2}\left(b_{2}^{i}\right)=\sum_{q=2 n-k+2}^{2 n} \sum_{m, l ; 2 l+m=q}\left(\frac{\partial H}{\partial b_{2}^{i}}+\frac{\partial H}{\partial W_{2}} \frac{\partial W_{2}}{\partial b_{2}^{i}}\right) .
$$

Equation (53) implies that $f_{2 n-k+2}^{\prime}\left(b_{2}^{i}\right)$

$$
=\sum_{q=2 n-k+2}^{2 n} \sum_{2 l+m=q}\left(\frac{\partial H}{\partial W_{2}} \frac{\partial^{2} W_{2}}{\partial b_{2}^{i 2}}+\frac{\partial^{2} H}{\partial b_{1}^{i 2}}+2 \frac{\partial H}{\partial b_{2}^{i}} \frac{\partial H}{\partial W_{2}} \frac{\partial W_{2}}{\partial b_{2}^{i}}+\frac{\partial^{2} H}{\partial W_{2}^{2}}\left(\frac{\partial W_{2}}{\partial b_{2}^{i}}\right)^{2}\right)
$$

and it is now apparent from equations (31), (52) and (54) that assumption $A$ is satisfied if and only if $\frac{\partial^{2} E \pi \mid \beta}{\partial b_{2}^{2}}<0$ for all $b_{2}$ such that $0 \leq b_{2} \leq \bar{v}$. (E$\pi^{i}$ is strictly 
concave in $b_{2}^{i}$ when all demanders besides bidder $i$ use the strategy $\beta$ ). We have now shown that $\beta$ is a best response to itself under conditions $\mathrm{A}$ and $\mathrm{B}$.

Lemma 10. If all $n$ players besides bidder $i$ use a bidding function that is type $M$ and undominated, then $f_{v}(x)>0$ for $0 \leq x \leq B_{2}(\bar{v}, \bar{v})$.

Proof: Recall that $F_{v}$ equals Prob(At least $l$ bidders make 2 bids that are less than or equal to $x$, at least $m+l$ bidders make at least 1 bid that is less than or equal to $x$ ).

The probability that a randomly chosen bidder makes 2 bids that are less than or equal to $x$ is given by:

$$
\int_{0}^{x} \int_{0}^{\bar{v}} g\left(v_{1}, v_{2}\right) d v_{2} d v_{1}
$$

The term in (55) follows from the fact that if $B$ is undominated, that $v_{1}^{y} \geq v_{2}^{y} \geq$ $B_{2}\left(v_{1}^{y}, v_{2}^{y}\right)$ (bidding an amount higher than one's valuation is dominated). The term is clearly strictly increasing in $x$ if $B$ is type $M$ and undominated for $x$ such that $0 \leq x \leq \bar{v}$. Now consider the probability that a randomly chosen bidder makes at least one bid that is less than or equal to $x$. The probability equals:

$$
1-\int_{B_{2}^{-1}\left(v_{2} \mid v_{1}=x\right)}^{\bar{v}} \int_{B_{2}^{-1}\left(v_{2} \mid v_{1}=x\right)}^{\bar{v}} g\left(v_{1}, v_{2}\right) d v_{2} d v_{1} \text {. }
$$

The last term results from the fact that underbidding on the higher valued unit is dominated by bidding an amount equal to the higher valuation and from the fact that $v_{1}^{y} \geq v_{2}^{y}$. This last equation is also strictly increasing in $x$ for all $x$ such that $0 \leq x \leq \bar{v}$. It follows that $F_{v}(x)$ is strictly increasing in $x$ and that $f_{v}(x)>0$; $0 \leq x \leq B_{2}(\bar{v}, \bar{v})$.

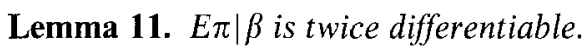

Proof: By assumption $\gamma \in C^{2}$. It follows that $g\left(v_{1}, v_{2}\right) \in C^{1}$, since $g\left(v_{1}, v_{2}\right)=$ $2 \gamma\left(v_{2}\right) d \gamma\left(v_{2}\right)\left(\gamma\left(v_{1}\right)-\gamma\left(v_{2}\right)\right) d \gamma\left(v_{1}\right)$. Since $g\left(v_{1}, v_{2}\right) \in C^{1}, \int_{0}^{x} \int_{0}^{y} g\left(v_{1}, v_{2}\right) d v_{2} d v_{1}$ is twice differentiable with respect to $x$ and $y$ and thus continuous in $x$ and $y$. It follows that $H(x, y, G, n, m, l)$, the product of twice differentiable functions, is twice differentiable with respect to $x$ and $y$, and thus continuous in $x$ and $y$. Therefore, $F_{v}(x)=$ $\sum_{q=v}^{2 n} \sum_{l, m ; 2 l+m=q} H\left(x, \beta^{-1}(x), G, n, m, l\right)$ is twice differentiable in $x$. This implies that $f_{\nu}(x)$ is differentiable and continuous in $x$.

$E \pi^{i}\left(b_{1}^{i}, b_{2}^{i}\right)$ is differentiable in $b_{1}^{i}$ and $b_{2}^{i}$ because an anti-derivative of a continuous function is differentiable. Clearly therefore, the first derivatives are differentiable in $b_{1}^{i}$ and $b_{2}^{i}$. We have now shown that $E \pi^{i}\left(b_{1}^{i}, b_{2}^{i}\right)$ is twice differentiable.

\section{References}

Forsythe, R., Isaac, M.: Demand revealing mechanisms for private good auctions. Res. in Exp. Econ. 2, 45-61 (1982)

Vickrey, W.: Counterspeculation, auctions, and competitive sealed tenders. J. Finance 16, 8-37 (1961)

Weber, R.: Multiple-object auctions. In: Auctions, bidding and contracting, pp. 165-190. New York:

New York University Press 1983 